\title{
Increased complexity of Tmem16a/Anoctamin 1 transcript alternative splicing
}

\author{
Kate E O'Driscoll, Rachel A Pipe and Fiona C Britton ${ }^{*}$
}

\begin{abstract}
Background: TMEM16A (Anoctamin 1; ANO1) is an eight transmembrane protein that functions as a calciumactivated chloride channel. TMEM16A in human exhibits alternatively spliced exons (6b, 13 and 15), which confer important roles in the regulation of channel function. Mouse Tmem16a is reported to consist of 25 exons that code for a 956 amino acid protein. In this study our aim was to provide details of mouse Tmem16a genomic structure and to investigate if Tmem16a transcript undergoes alternative splicing to generate channel diversity.

Results: We identified Tmem16a transcript variants consisting of alternative exons 6b, 10, 13, 14, 15 and 18. Our findings indicate that many of these exons are expressed in various combinations and that these splicing events are mostly conserved between mouse and human. In addition, we confirmed the expression of these exon variants in other mouse tissues. Additional splicing events were identified including a novel conserved exon 13b, tandem splice sites of exon 1 and 21 and two intron retention events.

Conclusion: Our results suggest that Tmem16a gene is significantly more complex than previously described. The complexity is especially evident in the region spanning exons 6 through 16 where a number of the alternative splicing events are thought to affect calcium sensitivity, voltage dependence and the kinetics of activation and deactivation of this calcium-activated chloride channel. The identification of multiple Tmem16a splice variants suggests that alternative splicing is an exquisite mechanism that operates to diversify TMEM16A channel function in both physiological and pathophysiological conditions.
\end{abstract}

\section{Background}

Alternative splicing of pre-mRNAs is a powerful regulatory mechanism that can increase mRNA transcript variety and effect functional diversification of proteins [1]. Within the cardiovascular system, alternative splicing affects cardiac function by regulating proteins involved in cellular excitation, including ion channels [2-8].

Calcium-activated chloride currents have been recorded in cardiac muscle cells from various species including mouse [9], and play an important role in the cardiac action potential [10-12]. In 2008, three independent groups identified Tmem16a as a strong candidate gene to encode (or at least a major component of) a calcium-activated chloride channel [13-15]. Tmem16a belongs to a family of ten mammalian paralogs (Tmem16 (a-h, j-k)) that are highly conserved membrane spanning proteins. In recombinant expression systems, Tmem16a (or Ano1) and Tmem16b

\footnotetext{
* Correspondence: fbritton@medicine.nevada.edu

Department of Physiology and Cell Biology, 1664 North Virginia Street, University of Nevada School of Medicine, Reno, Nevada 89557-0046, USA
}

(or Ano2) generate calcium-activated chloride currents [13-18] with similar biophysical and pharmacological properties to currents recorded from native tissues [19]. We and others have identified Tmem16a expression in mouse and human heart [20,21].

The human TMEM16A gene exhibits three alternatively spliced exons (6b, 13 and 15) as well as an alternative transcription start site [13]. Ferrera et al. reported that the biophysical properties of human TMEM16A are regulated by alternative splicing and TMEM16A splice variants form functional channels that display different properties [22]. The alternative exon $6 \mathrm{~b}$ (encoding 22 amino acids) may play an important role in the regulation of the TMEM16A channel by calcium, since exclusion of this exon increases the calcium sensitivity of the channel $\sim 4$-fold [22]. Exon 13 , encoding 4 amino acids, contributes significantly to TMEM16A channel kinetics, since exclusion of this exon significantly reduces the voltage dependence of activation [22]. A recent study showed that exon 15 (encoding 26 amino acids) exclusion results in significantly faster 
activation and deactivation kinetics [23]. In addition, Mazzone et al, showed significant differences in expression of alternatively spliced TMEM16A exons in patients with diabetic gastroparesis when compared to non-diabetic controls [23]. Therefore, it appears that alternative splicing of human TMEM16A plays an important role in the regulation of calcium-activated chloride channel function.

The reported mouse Tmem16a gene [GenBank: NC 000073] is composed of 25 exons that code for a 956 amino acid protein [GenBank: NP_848757]. Unlike human, mouse Tmem16a, as annotated, does not contain alternative exons $6 \mathrm{~b}, 13$ or 15 . We and others however, have reported that Tmem16a transcripts containing these alternative exons are expressed in mouse stomach, intestine [24] and vascular [25] smooth muscle tissues. It is likely that alternative splicing of Tmem16a transcript in mouse may lead to a number of different TMEM16A channel proteins with altered biophysical properties similar to human TMEM16A [22,23]. In this study our aim was to provide detailed information of the structure of the mouse Tmem16a gene and to investigate if Tmem16a transcript undergoes alternative splicing to generate channel diversity in mouse heart. This study demonstrates that the structure of Tmem16a gene is significantly more complex than previously indicated. The complexity is especially evident in the region containing exons 6 through 16. Determining the variation of Tmem16a transcript expression in heart is an important foundation for future studies of the physiological role of Tmem16a channels in heart.

\section{Methods}

RNA isolation and RT-PCR

Total RNA was isolated from mouse tissues using TRIzol reagent (Invitrogen, Carlsbad, CA). Human heart RNA was purchased from Agilent Technologies (Santa Clara, CA). First-strand cDNA was prepared from $1 \mu \mathrm{g}$ of RNA using oligo $(\mathrm{dT})_{(12-18)}$ primer and Superscript II reverse transcriptase (Invitrogen). AmpliTaq Gold ${ }^{\circledR}$ PCR reagent (Applied Biosystems, Foster City, CA) was used to amplify each of the Tmem16 paralogs and Tmem16a splice variants. PCR primers were designed using the mouse and human Tmem16a mRNA sequences [GenBank: NM_178642 and GenBank: NM_018043]. Details of the primer sets used are provided in additional file 1 , Table S1 and Table S2). Glyceraldehyde-3-phosphate dehydrogenase (GAPDH) was used as a control for cDNA integrity. No template PCR reactions served as controls for primer contamination. PCRs were performed in a 2720 Thermal Cycler (Applied Biosystems). Amplification consisted of $95^{\circ} \mathrm{C}$ for $10 \mathrm{~min}$, then 35 cycles of $95^{\circ} \mathrm{C}$ for $15 \mathrm{sec}, \mathrm{T}_{\mathrm{a}}$ for $20 \mathrm{sec}$ and $72^{\circ} \mathrm{C}$ for $30-60 \mathrm{sec}$, followed by a final step at $72^{\circ} \mathrm{C}$ for $7 \mathrm{~min}$.

\section{Splice variant identification, sequencing and bioinformatics}

PCR products were resolved on 2-3\% super fine agarose (Amresco, Solon, OH) gels along with a 100 bp molecular weight marker. Tmem16a amplification products were either purified (QIAquick Gel Extraction Kit, Qiagen, Valencia, CA) or TA cloned into the pcDNA3.1 vector (Invitrogen). All fragment sequencing was performed at the Nevada Genomics Center. Nucleotide and protein sequences were analyzed using Vector NTI version 11 software (Invitrogen) and BLASTN software at the National Center for Biotechnology Information (NCBI) database http://www.ncbi.nlm.nih.gov/BLAST/.

Transmembrane regions were predicted using the CBS TMHMM Server v 2.0 http://www.cbs.dtu.dk/services/ TMHMM/ and TMpred http://www.ch.embnet.org/software/TMPRED_form.html programs. The TAndem Splice Site DataBase (TassDB2) http://gen100.imb-jena. de/TassDB2/ was used to confirm alternative tandem splice sites in mouse and human Tmem16a. The Scansite database http://scansite.mit.edu/motifscan_id.phtml was used to identify motifs in the TMEM16A alternative splice variants.

\section{Results and Discussion}

\section{Tmem 16a genomic structure is highly conserved in} mouse and human

Genomic sequences for human [GenBank: NC_000011) and mouse [GenBank: NC_000073] Tmem16a were compared with their respective RNA sequence [GenBank: NM_018043 and NM_178642]. The reported mouse Tmem16a gene is composed of 25 exons, however, our analysis has revealed that mouse Tmem16a gene consists of at least 29 exons, ranging in size from 12 bp to 1621 bp. Table 1 provides details of mouse Tmem16a gene structure, including the number of exons, exon and intron size, 5' donor and 3'acceptor splice site sequences. The exon sizes of human TMEM16A are also provided for comparison purposes. The exon-intron junctions of Tmem16a comply with the consensus dinucleotide GTAG rule. The number and size of each exon in mouse Tmem16a gene are highly conserved with human TMEM16A. There is some divergence between mouse and human Tmem16a genes at the 5' and 3' termini. For example, in mouse, exon 1 and exon $1 \mathrm{~b}$ differ from human. The putative translational initiation codon of mouse and human Tmem16a are located in exon 1b and exon 1 respectively. The nucleotides adjacent to the initiator codons (mouse; GCCACCATGA and human; GCCACGATGA), constitute a favorable Kozak consensus initiation site [26]. Human TMEM16A has been reported to exhibit alternative splicing [13]. This study indicates that Tmem16a in mouse also exhibits multiple 
Table 1 Genomic Organization of Tmem16a

\begin{tabular}{|c|c|c|c|c|c|}
\hline \multicolumn{2}{|c|}{ Human } & \multicolumn{4}{|c|}{ Mouse } \\
\hline $\begin{array}{l}\text { Exon } \\
\text { number }\end{array}$ & $\begin{array}{c}\text { Exon size } \\
\text { (bp) }\end{array}$ & $\begin{array}{c}\text { Exon } \\
\text { number }\end{array}$ & $\begin{array}{c}\text { Exon size } \\
\text { (bp) }\end{array}$ & 3' Acceptor and 5' Donor splice site sequences & $\begin{array}{c}\text { Intron } \\
\text { size (bp) }\end{array}$ \\
\hline \multirow[t]{2}{*}{1} & 413 & 1 & 119 & CAGGTG gtagga & $\sim 59,580$ \\
\hline & & $1 b$ & 146 & 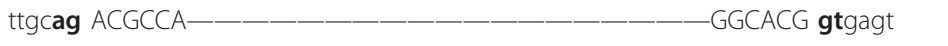 & 8,958 \\
\hline 2 & 333 & 2 & 333 & ——GAGGAT gtgagt & 12,229 \\
\hline 3 & 99 & 3 & 99 & ctctag ACCAAA_————_- & 1,264 \\
\hline 4 & 152 & 4 & 152 & ccacag GTGTAC _ _ _ _ _ _ & 1,340 \\
\hline 5 & 55 & 5 & 55 & ctgcag ATTCGA__—__- & 3,649 \\
\hline 6 & 52 & 6 & 52 & —_GCATGG gtaagg & 1,862 \\
\hline $6 b$ & 66 & $6 b$ & 66 & _-AATACG gtaaga & 495 \\
\hline 7 & 56 & 7 & 56 & _—_—_CACGAT gtaagt & 3,274 \\
\hline 8 & 42 & 8 & 42 & tcacag GGGGAC - — — - & 6,143 \\
\hline 9 & 65 & 9 & 65 & ——_GGTCAG gtaagt & 1,290 \\
\hline 10 & 135 & 10 & 135 & gcatag GAAATA-_- - - - - CCCCAG gtaggc & 3,318 \\
\hline 11 & 161 & 11 & 161 & ttacag TATGGA_—_- & 11,845 \\
\hline 12 & 83 & 12 & 83 & ctacag CTGCCA-———- & 2,181 \\
\hline \multirow[t]{2}{*}{13} & 12 & 13 & 12 & ccgcag GAAGCT_———— & 161 \\
\hline & & $13 b$ & 120 & ttccag $\mathrm{CACCT}-\ldots$ & 273 \\
\hline 14 & 72 & 14 & 72 & tctcag GATCAT_- ——_- - - & 2,874 \\
\hline 15 & 78 & 15 & 78 & - - AAATTG gtactt & 972 \\
\hline 16 & 75 & 16 & 75 & $\operatorname{ttg}$ cag ACCGAC - - - - - - - - & 3,034 \\
\hline 17 & 202 & 17 & 202 & ————AGATTG gtgagt & 167 \\
\hline 18 & 112 & 18 & 112 & cctcag AGGTCC-————- & 434 \\
\hline 19 & 58 & 19 & 58 & tcccag GTTGT_————- & 1,892 \\
\hline 20 & 101 & 20 & 101 & ctacag TGTGCC-————————- & 786 \\
\hline 21 & 146 & 21 & 146 & ctgcag GAAGAT_- - - - - - & 4,324 \\
\hline 22 & 153 & 22 & 153 & ctgcag TCATTC————- & 6,207 \\
\hline 23 & 53 & 23 & 53 & ctctag GCATCT-———-_- - - - & 1,554 \\
\hline 24 & 185 & 24 & 185 & tggcag GCCTIT——————————————————CTGCAG gtacta & 2,635 \\
\hline 25 & 106 & 25 & 106 & 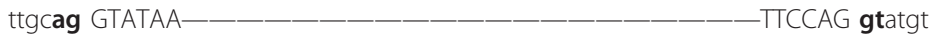 & 2,435 \\
\hline 26 & 1809 & 26 & 1621 & ctgcag AACCTG & \\
\hline
\end{tabular}

Intron size refers to the intron following the exon listed in each row.

alternative exons, which generates numerous isoforms through alternative splicing of the primary transcript. A close examination of the mouse Tmem16a genomic sequence revealed that similar sequences to these human exon variants (exons 6b, 13 and 15) could be identified (Table 1). Additionally, a number of novel splicing events were identified (Table 1) and the details of these transcript variants are outlined throughout this report.

\section{Identification of Tmem 16a splice variant expression in mouse heart}

RT-PCR was initially used to identify the expression of Tmem16 paralogs in mouse heart using primers specific for each of the Tmem16 family members (Additional file 1, Table S1). In addition to Tmem16a, several other Tmem16 transcripts were detected in mouse heart: Tmem16c, $d, e, f, h, j$ and $k$ (Figure 1A). The expression of Tmem16b and Tmem16g transcripts were not detected.
To determine if alternative exons, homologous to the exons 6b, 13 and 15 of human TMEM16A [13], are also expressed in mouse heart we performed RT-PCR analysis of Tmem16a (Figure 1B). Primer sets \pm exon $6 \mathrm{~b}, \pm$ exon 13 and \pm exon 15 amplify fragments that differ in size ( $\pm 66 \mathrm{bp}, 12 \mathrm{bp}$ and $78 \mathrm{bp}$, respectively) corresponding to the size of the alternative exon. The amplification of two fragments using \pm exon $6 \mathrm{~b}$ and \pm exon 15 primers clearly indicates that Tmem16a variants that either include or exclude these alternative exons are expressed in mouse heart (Figure 1B). The presence or absence of the alternative 12 bp micro-exon 13 is difficult to resolve by gel electrophoresis, therefore additional primers were designed that either hybridize within each of the alternative exons to specifically amplify them or that span bordering exons to exclude them (Figure $1 \mathrm{C}$ and Additional file 1, Table S2). For example, Tmem16a transcripts lacking exon 15 (-exon 15) were selectively amplified using a primer that 


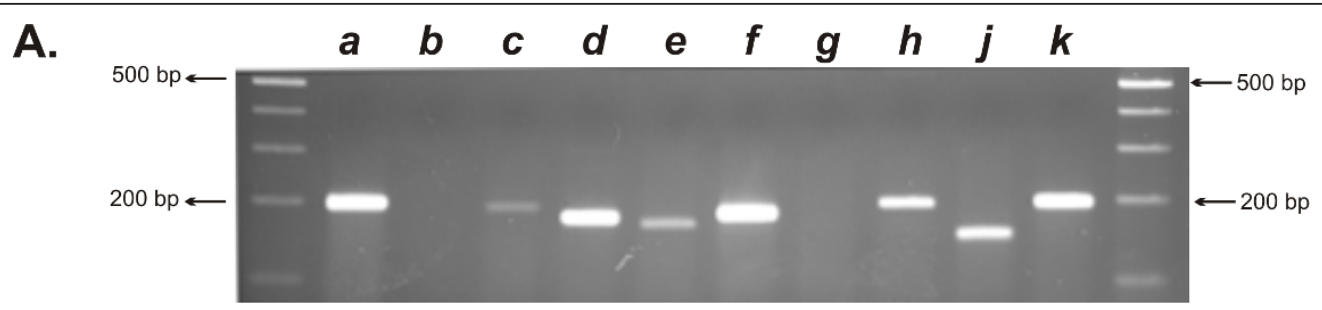

B.
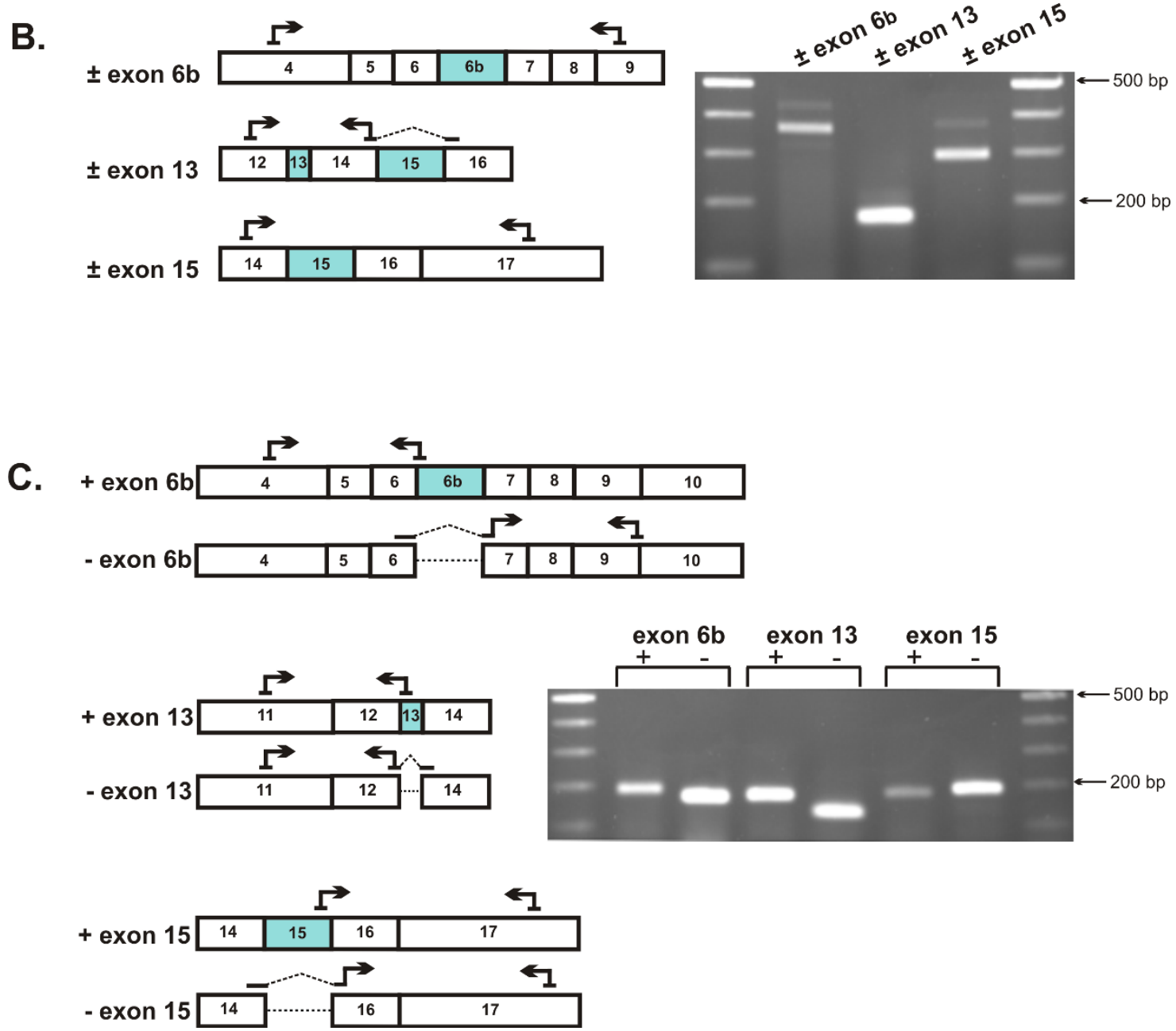

Figure 1 Identification of alternative Tmem $16 a$ exons 6 b, 13 and 15 in mouse heart. A. Expression of Tmem 16 family members were analyzed in mouse heart using RT-PCR primers specific for each of the ten Tmem 16 paralogs. Amplification products were resolved on $2 \%$ agarose gels alongside a $100 \mathrm{bp}$ marker. Fragments of the expected molecular size indicated the expression of Tmem $16 a, c, d, e, f, h, j$ and $k$ paralogs in mouse heart. Tmem $16 b$ and $g$ were not detected. B. Schematic representations of regions of Tmem $16 a$ RNA. Boxed numbers indicate exons relative to the Tmem16a gene. Alternative exon $6 \mathrm{~b}(66 \mathrm{bp})$, exon 13 (12 bp) and exon 15 (78 bp) in the mouse Tmem16a coding sequence are highlighted. RT-PCR primers ( \pm exon $6 \mathrm{~b}, \pm$ exon $13, \pm$ exon 15 ), which amplify across each alternative exon are indicated. RT-PCR gel analysis of exon 6b, 13 and 15 Tmem16a variants expressed in mouse heart. Primers \pm exon 6b amplify a 367 and/or a 433 bp product, primers \pm exon 13 amplify a 165 and/or a 177 bp product, primers \pm exon 15 amplify a 297 and/or a 375 bp product. The upper bands correspond to Tmem $16 a$ variants that include the alternative exon and differ in size corresponding to the size of that exon. C. RT-PCR primer sets that span exon boundaries to selectively amplify individual alternative exons are indicated. Gel analysis of Tmem16a fragments expressed in mouse heart that either include or exclude exons $6 b, 13$ or 15. Each primer set yielded a single band of the expected size. RT-PCR products were sequenced for confirmation. 
spans the junction of exons 14 and 16. Similarly, Tmem16a transcripts containing exon 15 (+exon 15) were specifically amplified using a primer internal to exon 15 . RT-PCR gel analysis (Figure 1C) and fragment sequencing confirmed that Tmem16a variants are expressed in mouse heart that either exclude or include exon 6b, exon 13 and exon 15, identical to those we identified in mouse gastrointestinal tissues [24].

A comparison of the amino acid sequences of mouse and human alternative exons show a high similarity; the four amino acids (EAVK) of exon 13 are $100 \%$ identical, the 22 and 26 amino acids encoded by exon $6 \mathrm{~b}$ and exon 15 , share $95.5 \%$ and $80.8 \%$ identity, respectively. Considering the high percentage amino acid identity of these exon between the two species, we can expect that the regulatory properties attributed to these channel domains in human [22,23], will most likely confer similar functional roles in the mouse, in that, exon $6 \mathrm{~b}$ may confer calcium sensitivity, exon 13 may contribute to voltage dependence and exon 15 may play a role in the activation and deactivation kinetics of this calcium activated chloride channel. The inclusion of alternative exons $6 \mathrm{~b}, 13$ and 15 would result in additional amino acids in intracellular channel domains and are not predicted to alter the eight transmembrane topology of the TMEM16A protein.

\section{Tmem 16a transcripts with combinations of alternative exons $6 b, 13$ and 15 create diversity}

A detailed investigation into the expression of various alternative exon combinations has not been reported for Tmem16a. To explore if different combinations of these alternative exons exist in mouse and human heart, we employed RT-PCR analysis to examine the region of the Tmem16a transcript encompassing alternative exons $6 \mathrm{~b}$, 13 and 15 (Figure 2). Eight Tmem16a variants may be generated due to alternative combinations of exon inclusion or exclusion (Figure 2A). RT-PCR analysis generated Tmem16a transcripts of various molecular sizes expressed in mouse and human heart (Figure 2B), corresponding to different combinations of Tmem $16 a$ alternative exon 6b, 13 or 15 inclusion or exclusion. The complexity of alternative exon combinations is difficult to resolve by size analysis alone, largely due to the micro exon 13 and two possible fragments of the same size (Figure 2A). Thus, the entire RT-PCR amplifications were cloned, and sequence analysis of multiple clones confirmed that at least six distinct Tmem16a splice variants consisting of various combinations of exons 6b, 13 and 15 are expressed in mouse and human heart.

Both mouse and human heart express the following three exon combinations: Tmem16a $(-6 \mathrm{~b},+13,-15)$, Tmem16a $(+6 \mathrm{~b},+13,-15)$, and Tmem16a $(-6 \mathrm{~b},+13,+15)$. We did not identify Tmem16a transcripts consisting of exon $6 \mathrm{~b}$ alone $(+6 \mathrm{~b},-13,-15)$ or a Tmem $16 a$ variant containing $6 \mathrm{~b}$ and 15 but lacking exon $13(+6 \mathrm{~b},-13,+15)$ in either mouse or human heart. It appears, at least in heart, that exon $6 \mathrm{~b}$ is only expressed in the presence of exon 13 . However, we did identify Tmem16a transcripts containing exon 15 in the absence of both exons $6 \mathrm{~b}$ and 13 (-6b, -13, +15 ) in mouse heart. Interestingly, a Tmem16a transcript missing all three exons $(-6 b,-13,-15)$ was only identified in mouse heart, in agreement with the reported Tmem16a [GenBank: NM_178642] and although a human transcript variant $2(-6 b,-13,-15)$ [GenBank: NR_030691] exists we did not confirm its presence in human heart. All human TMEM16A transcripts contained exon 13, in agreement with [GenBank: NM_018043] $(-6 b,+13,+15)$. The largest Tmem16a variant consisting of all three exons was confirmed by sequence analysis in human heart. Although this Tmem16a variant was not confirmed in our screening of mouse heart clones, we cannot rule out its existence since our gel analysis indicates a band close to the expected size of this variant, albeit much weaker than in human (Figure 2B). Indeed, it is possible that different mouse and human tissues may express different combinations of these alternative exons, however, quantification of our data would not provide more information since the tissues examined in this study contain a mixed population of cells. The deduced amino acid sequence of mouse TMEM16A containing alternative exons combinations (6b, 13 and 15) all read in-frame, indicating that these alternative exons are additional channel components similar to those of human TMEM16A [22]. BLASTn searches of dbEST and Refseq RNA databases identified a number of mouse ESTs containing exon $6 \mathrm{~b}$ and exon 13. Indeed, one mouse EST [GenBank: BI151426] was identified for the $+6 \mathrm{~b},+13,-15$ exon combination. EST supporting query sequences were identified for mouse [GenBank: BI685387] containing exon 13 alone $(-6 b,+13,-15)$. No ESTs were found in mouse containing both exon 13 and 15 , however this is the exon combination in human that corresponds to TMEM16A [GenBank: NM_018043].

In addition to alternative exons $6 \mathrm{~b}, 13$ and 15 , we also identified a number of novel Tmem16a splicing events in mouse and human heart; exon 10 and exon 14 exclusion, the inclusion of a novel exon, and two intron retention events.

\section{Novel Tmem $16 a$ transcripts with exon 10 or exon 14 exclusion}

We identified two previously unreported Tmem16a exon skipping events, exon 10 and exon 14, in mouse and human heart (Figure 3). Exclusion of exon 10 (135 bp) in Tmem16a RNA would result in an in-frame deletion of 45 amino acids in the channel protein. Exon 10 encodes a hydrophobic region predicted to be the first transmembrane spanning domain of TMEM16A. 


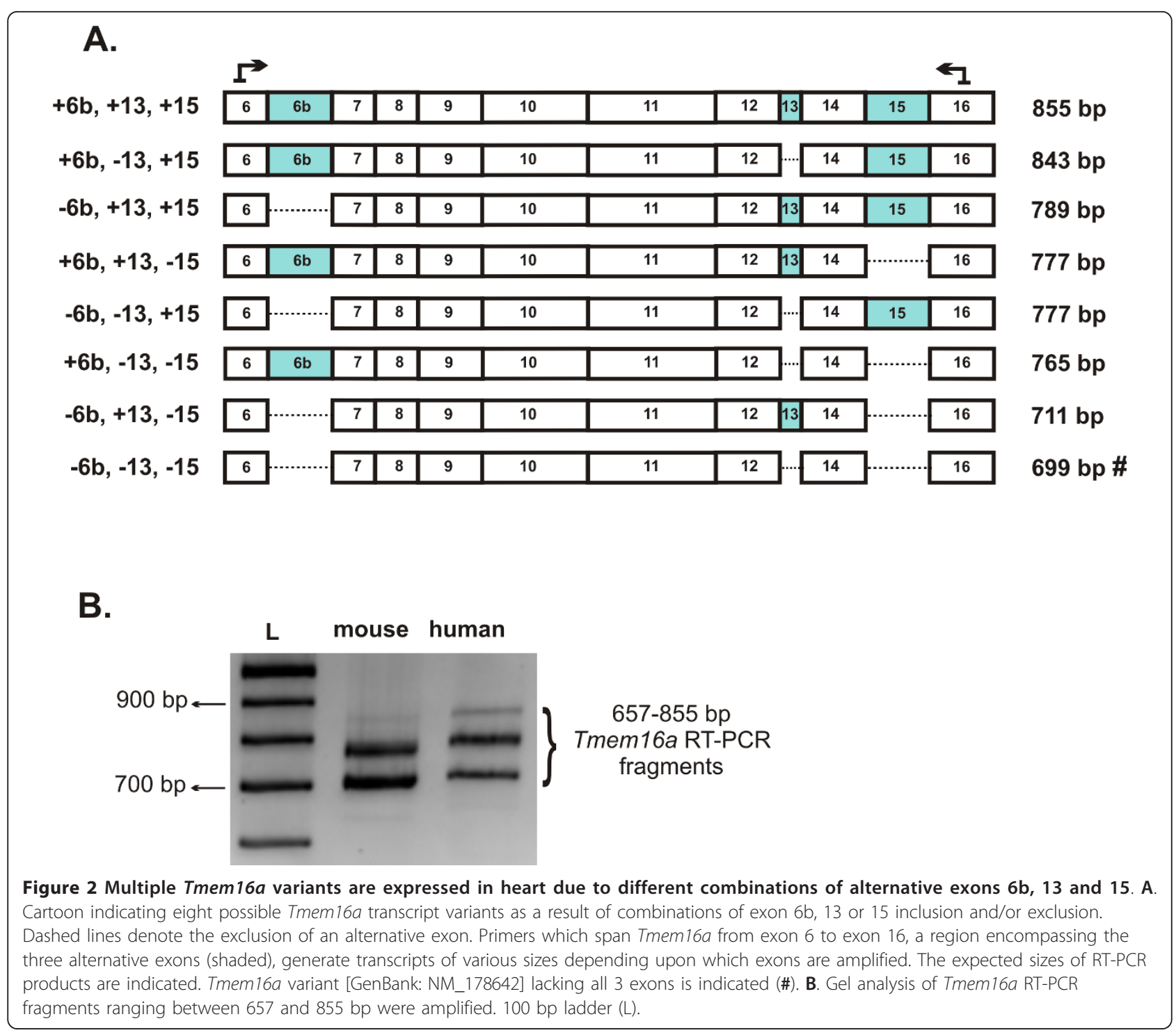

Therefore, exon 10 exclusion would result in a change in the transmembrane topology. We identified through sequencing two different Tmem16a (-10) transcripts, in that both transcripts lacked exon $6 \mathrm{~b}$ and included exon 13, however, they differed in whether or not exon 15 was included. This is highlighted in the cartoon in Figure 3A. We employed an additional primer set to specifically amplify Tmem16a (-10) variants (Figure 3A) and sequence analysis confirmed the presence of these transcript variants in mouse and human heart.

Sequence analysis revealed that the Tmem16a (-14) variant included exons $6 \mathrm{~b}$ and 13 and excluded exon 15 (Figure 3B). Exclusion of exon 14 (72 bp) in Tmem16a RNA would result in an in-frame deletion of 24 amino acids in the first intracellular loop of the TMEM16A channel. A PKC site is present in exon 14 at position S462 [GenBank: NP_848757] and one could speculate that Tmem16a transcripts lacking exon 14 may encode channels that differ in modulation by PKC. Likewise, the expression of this variant was confirmed in mouse and human heart using primers which specifically amplify Tmem16a (-14) variants.

\section{Tmem 16a transcripts with a novel conserved exon 13b}

Sequence screening of our Tmem16a clone library, identified an unusual Tmem16a transcript that excluded alternative exons $6 \mathrm{~b}, 13$ and 15 yet contained an additional 120 nucleotides between exon 12 and exon 14 . Evaluation of Tmem16a genomic sequence revealed that these 120 nucleotides are located within the $554 \mathrm{bp}$ intronic region between exon 13 and 14 (Figure 4A) and are bordered by conserved 'GT-AG' acceptor and donor splice sites [27]. We assigned the additional sequence in this Tmem16a transcript as exon 13b. A close 


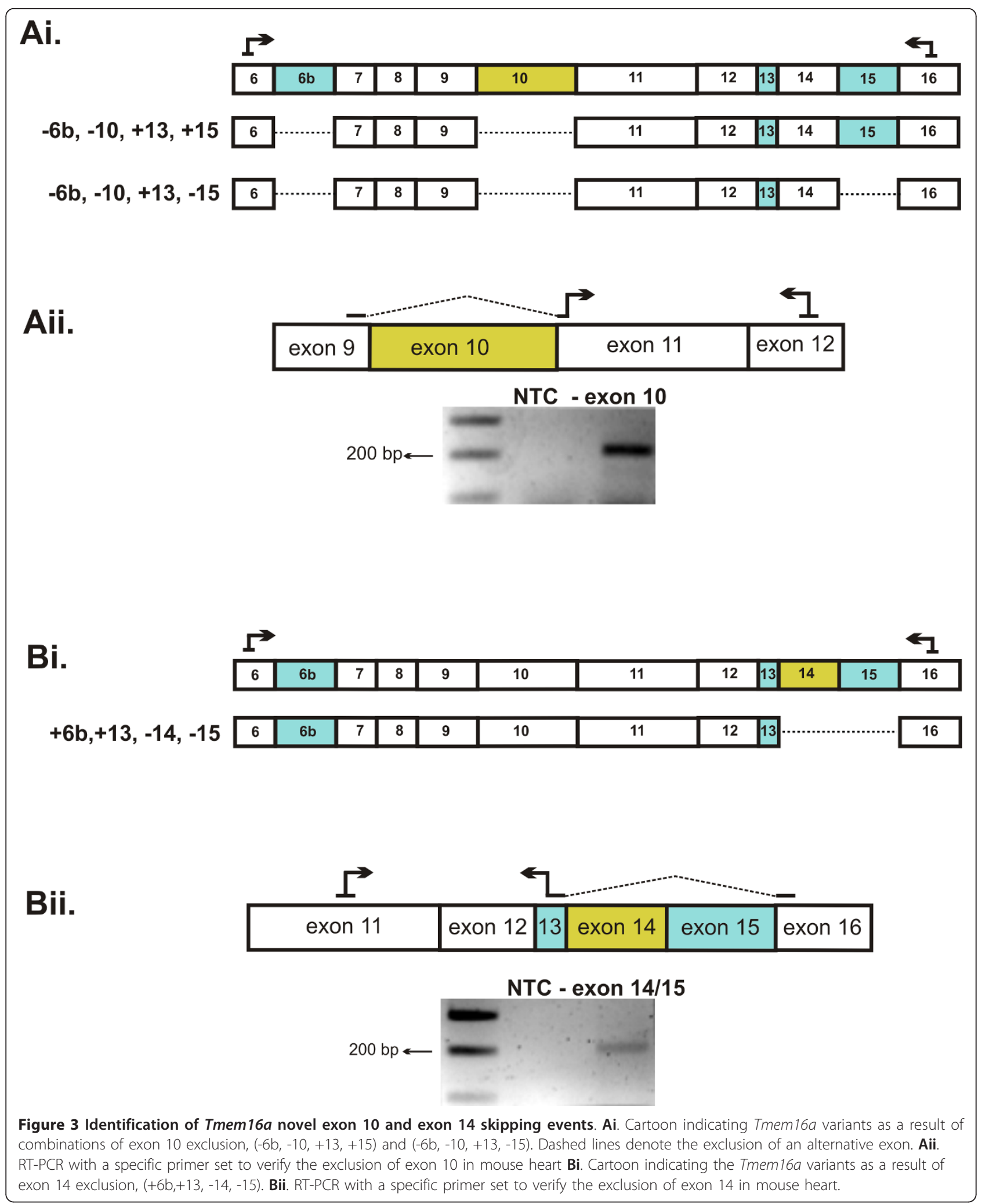




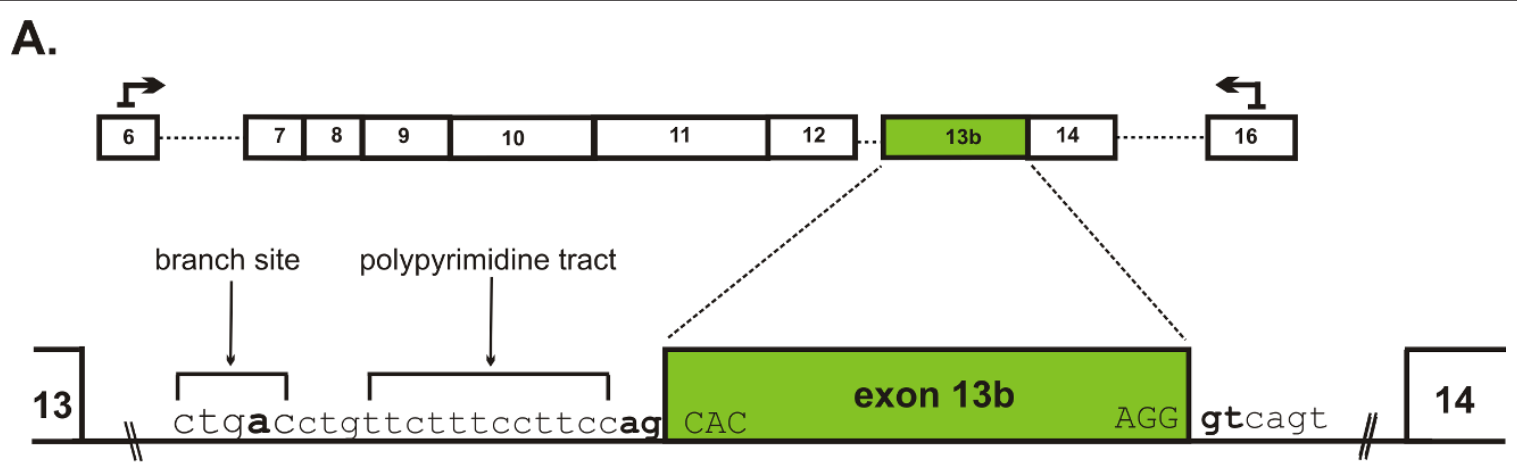

B.
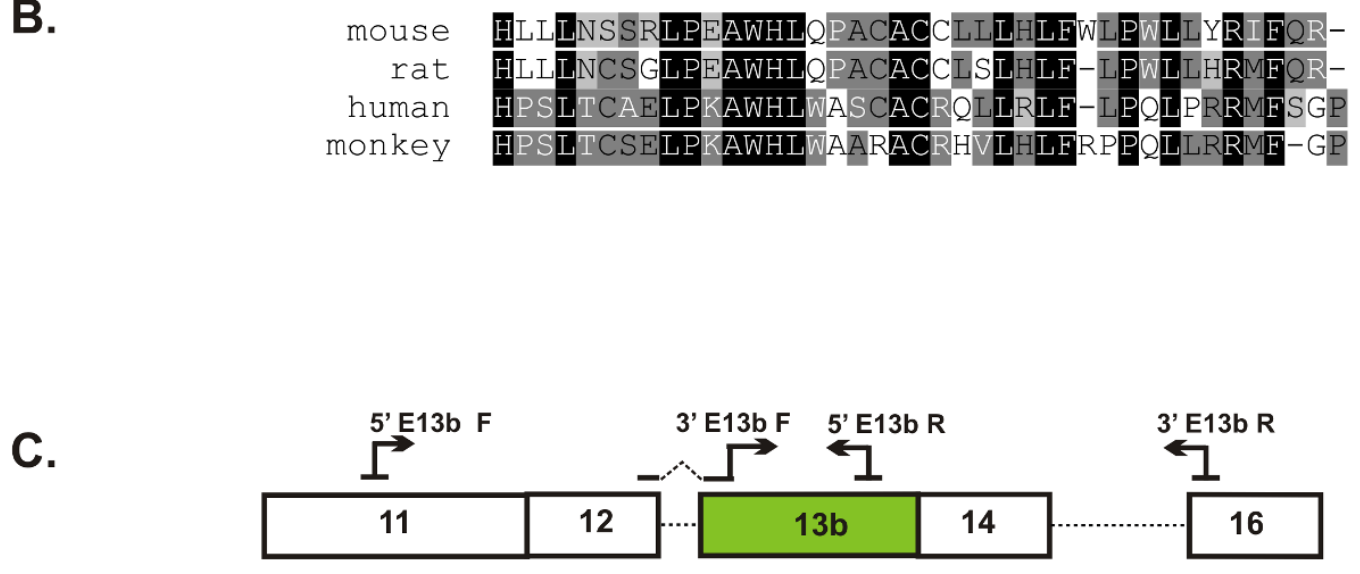

\section{5'Exon 13b}

\begin{tabular}{|c|c|c|c|}
\hline 11 & 12 & $13 b$ & 290 bp \\
\hline 11 & 12 & & $13 \mathrm{~b}$ \\
\hline
\end{tabular}

3'Exon 13b
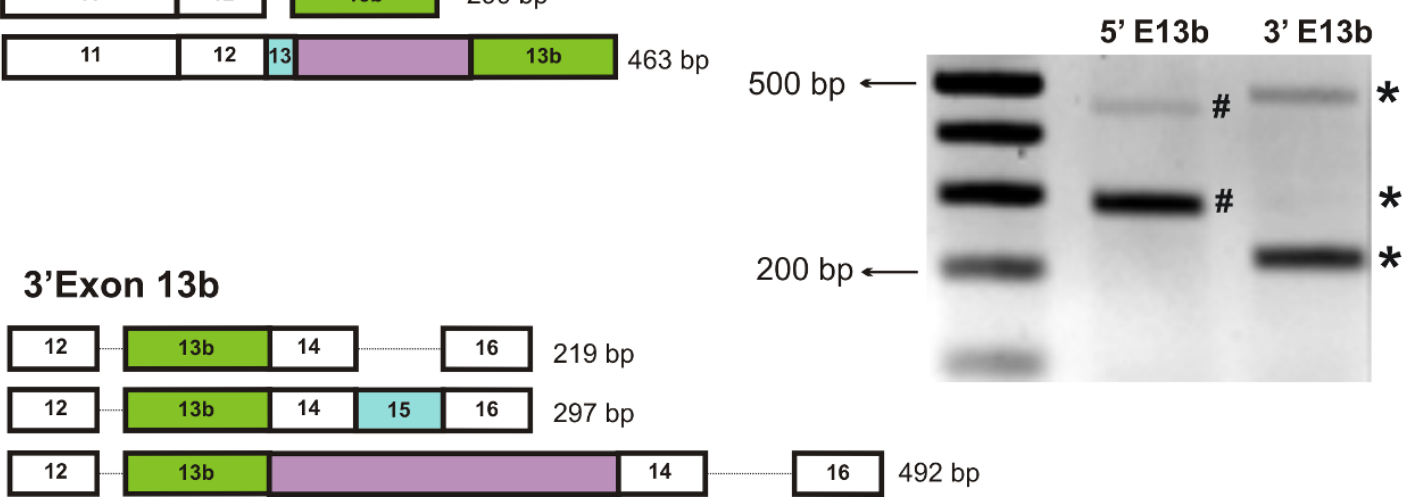

\begin{tabular}{|c|c|c|c|}
\hline $13 b$ & 14 & & \multicolumn{1}{|c|}{16} \\
\hline $13 b$ & 14 & 15 & 16 \\
\hline
\end{tabular}

$219 \mathrm{bp}$

$297 \mathrm{bp}$

Figure 4 Identification of a novel exon 13b in Tmem16a. A. Cartoon representation of the novel exon 13b identified through sequence analysis of a Tmem16a fragment amplified using primers that span exons 6 through 16. Exon13b (120 bp) is highlighted. The genomic region between exon 13 and 14 is expanded to indicate the location of exon 13b, the splice and branch sites and the polypyrimidine tract. B. Alignment of the 40 amino acids encoded by exon 13b of mouse with the corresponding residues identified in rat, human and rhesus monkey. C. RT-PCR with primer sets 5'Exon 13b and 3'Exon13b verified the expression of exon 13b in mouse heart. Cartoon of the amplification products generated with these primers is shown. Primer set 5'Exon13b produced two fragments, the 290 bp fragment consisted of exons 11,12 and $13 \mathrm{~b}$; the $463 \mathrm{bp}$ fragment consisted of exons 11, 12, 13 and13b plus the entire intronic sequence between exon 13 and $13 \mathrm{~b}$. The $3{ }^{\prime} \mathrm{E} 13 \mathrm{~b}$ primer set yielded three fragments. The $219 \mathrm{bp}$ and $297 \mathrm{bp}$ fragments included exons 12, 13b, 14, \pm 15, 16, and the 492 bp fragment consisted of 12, 13b, $14,-15,16$ plus the intronic sequence between exon $13 \mathrm{~b}$ and 14 . The intron retention events are highlighted. 
examination of the intronic sequence immediately upstream of exon 13b identified a conserved mammalian "CURAY" branchpoint sequence 18 nucleotides upstream of the 3' splice site (Figure 4A). The branchpoint (A nucleotide) is generally situated 18 to 40 nucleotides upstream of the 3' splice site and plays an important role in identifying this splice site $[28,29]$. An adjacent polypyrimidine tract, which increases the efficiency of branchpoint utilization [30], was also identified in this region (Figure 4A). Together, these findings add support that this $120 \mathrm{bp}$ sequence is a true exon. Inclusion of exon $13 \mathrm{~b}$ in the Tmem16a transcript would result in an inframe addition of 40 amino acids in TMEM16A. This region is rich in hydrophobic leucine residues (12/40 residues being leucines). It is not surprising then that hydropathy analysis suggests that exon 13b inclusion may constitute an additional domain in the TMEM16A channel integral to the membrane.

Considering that the exon structures of Tmem16a are highly conserved amongst homologs, we investigated if similar novel exons could be identified in other species. A close examination of the genomic Tmem16a sequence from rat, human and rhesus monkey [GenBank: NC_005100, GenBank: NC_000011, GenBank: NC_007871,] revealed that similar sequences to mouse Tmem16a exon 13b, bordered by conserved splice sites could be identified. An alignment of the corresponding amino acid sequence of these four species (Figure 4B) demonstrates $97.6 \%$ overall consensus with an identity of $41.5 \%$. Specific primers designed to amplify this novel exon in human heart were unable to identify its expression. Whether or not this exon is present in other human tissue types is unknown.

The expression of the novel exon 13b in mouse heart was investigated further using primer sets to specifically amplify the 5' and 3' regions surrounding the $120 \mathrm{bp}$ exon (Figure 4C). Sequence analysis revealed some interesting observations. First, we confirmed that exon 13b is utilized in mouse heart. Secondly, exon 13b is present in several Tmem16a transcripts that differ in whether they also include or exclude alternative exons 13 or 15 . Third, retention of the introns surrounding exon 13b increases the complexity of splicing in this region of Tmem16a transcript. RT-PCR with the primer set $5{ }^{\prime}$ Exon $13 \mathrm{~b}$, in which primers bind within exon 11 and 13b produced two amplification products (Figure 4C). Sequencing analysis indicated one Tmem16a fragment consisted of exons 11, 12 and 13b and the other fragment consisted of exons 11, 12, 13, 13b plus the entire $161 \mathrm{bp}$ intronic sequence between exon 13 and $13 \mathrm{~b}$ (Figure $4 \mathrm{C}$ ). The 3'Exon13b primer set, in which the primers hybridize across exon 12/13b junction and within exon 16, yielded three Tmem16a transcript fragments (Figure 4C). Two fragments differ in exon 15 inclusion/exclusion exons 12,
$13 \mathrm{~b}, 14, \pm 15,16$, and an unexpected fragment consisting of exon 12,13b, 14, -15, 16 plus the entire intronic sequence (273 bp) between exon 13b and 14 (Figure 4C). Intron retention events at the $5^{\prime}$ and $3^{\prime}$ of exon $13 \mathrm{~b}$ are certainly the result of alternative Tmem16a splicing and not genomic contamination since the $5{ }^{\prime}$ Exon $13 \mathrm{~b}$ and 3'Exon13b primers span at least $14.4 \mathrm{~kb}$ and $4.4 \mathrm{~kb}$ of genomic sequence, respectively.

The intron retention events at the 5'and 3' of exon 13b would introduce a premature stop codon in the Tmem16a transcript, resulting in a truncated protein just after the "EAVK" amino acids encoded by exon 13. The complete retention of an intron in a mature transcript is one of the least expected forms of alternative splicing and may occur due to weak splice sites, cis-regulatory elements or short intron lengths [31]. Indeed, the introns between exons 13 and $13 \mathrm{~b}$ and between exon $13 \mathrm{~b}$ and 14 are two of the shortest in the Tmem16a genomic sequence (see Table 1). Intron retention can result in the insertion of a premature stop codon being introduced to the mature transcript which may then be degraded by nonsense mediated decay [32]. Indeed, decreasing the mRNA levels of certain genes can be the purpose of alternative splicing [32]. However, sometimes the retention of an intron in the coding region can have a biological effect. For example, an intron retaining isoform of the KCNMA1 gene, which encodes the large conductance calcium-activated potassium channel (BK), facilitates splice variant regulation which contributes to structural and functional diversity of BK channel proteins in hippocampal neurons [33,34].

\section{Tmem 16a transcript scanning assay reveals additional novel splice variants}

It is clear from our analysis that Tmem16a transcript undergoes extensive splicing within the region between exons 6 and 16. In addition, not all of these exon inclusion/exclusion combinations are consistent between mouse and human. Indeed splicing events occurring at other regions of Tmem16a transcript may have important functional implications. Thus, we profiled the entire Tmem16a using overlapping RT-PCR primers to determine whether additional Tmem16a RNA splicing events occur in mouse and human heart. The transcript scanning assay and sequencing analysis revealed additional novel Tmem16a variants. These variants included exon 18 exclusion in both mouse and human Tmem16a and two alternative 3' donor sites, $\Delta 3$ 'exon 1 and $\Delta 3$ 'exon 21, in mouse. Details for mouse and human Tmem16a are summarized in Tables 2 and 3.

Exon 18 is 112 nucleotides and the exclusion of this exon shifts the encoded mouse TMEM16A out of frame at amino acid I563 [GenBank: NP_848757], truncating the protein from 956 to 596 amino acids. Exon 18 encodes the fifth transmembrane spanning domain of TMEM16A [35] 
Table 2 Mouse Tmem16a transcript scanning assay

\begin{tabular}{|c|c|c|c|c|}
\hline $\begin{array}{l}\text { Primer } \\
\text { Set }\end{array}$ & $\begin{array}{l}\text { Oligonucleotid } \\
\text { sequences }\left(5^{\prime} \rightarrow 3^{\prime}\right)\end{array}$ & $\begin{array}{l}\text { Tmem 16a } \\
\text { region }\end{array}$ & $\begin{array}{l}\text { Size } \\
\text { (bp) }\end{array}$ & $\begin{array}{c}\text { Alternative } \\
\text { exon(s) identified }\end{array}$ \\
\hline \multirow[t]{2}{*}{$\bar{A}$} & GAGGGAACCTCTGCGGACCGA (sense) & Exons 1-3 & 613 & \\
\hline & GCCAGGGCGCATGGATCTTC (antisense) & & 610 & $\Delta 3^{\prime}$ Exon 1 \\
\hline \multirow[t]{2}{*}{ B } & GGCCTGGAGTTGGAGAATGACG (sense) & Exons 2-10 & 602 & \\
\hline & GTGTAGGCTCCAAGCCAGGCAA (antisense) & & 668 & + Exon $6 \mathrm{~b}$ \\
\hline \multirow[t]{8}{*}{ C } & GAACAACGTGCACCAAGGCCAAGTA (sense) & Exons 6-16 & 699 & \\
\hline & TGGTGAAATAGGCTGGGAATCGGTC (antisense) & & 711 & + Exon 13 \\
\hline & & & 777 & + Exon 15 \\
\hline & & & 777 & + Exons $6 \mathrm{~b}+13$ \\
\hline & & & 789 & + Exons $13+15$ \\
\hline & & & 819 & + Exon 13b \\
\hline & & & 654 & - Exons $10+13+15$ \\
\hline & & & 705 & - Exons $6 b+13-14$ \\
\hline \multirow[t]{3}{*}{$\mathrm{D}$} & TGGAGCACTGGAAACGGAAG (sense) & Exons $12-20$ & 625 & \\
\hline & ATACAGAGCTCCATGAGGCAGC (antisense) & & 637 & + Exon 13 \\
\hline & & & 715 & Exons $13+15$ \\
\hline \multirow[t]{3}{*}{ E } & ACCGACAAGGTGAAGCTGAC (sense) & Exons $16-22$ & 731 & \\
\hline & GACGCAACAAACAGGGTGAC (antisense) & & 619 & - Exon 18 \\
\hline & & & 623 & $\Delta 3^{\prime}$ Exon 21 \\
\hline \multirow[t]{2}{*}{ F } & GAAGTTACGGCTGCATTGC (sense) & Exons $17-24$ & 719 & \\
\hline & ATGTAGAGGTACACCAGGCGAG (antisense) & & & \\
\hline \multirow[t]{2}{*}{ G } & GCCTCATGGAGCTCTGTATCC (sense) & Exons 20-26 & 767 & \\
\hline & CACCCAGTCCACAAAGTCACTC (antisense) & & & \\
\hline \multirow[t]{2}{*}{$\mathrm{H}$} & TGGTATAACATCCTCAGAGGTG (sense) & Exons $23-26$ & 656 & \\
\hline & TCACTTGGTGATGGTCAGAA (antisense) & & & \\
\hline
\end{tabular}

Table 3 Human TMEM16A transcript scanning assay

\begin{tabular}{|c|c|c|c|c|}
\hline $\begin{array}{l}\text { Primer } \\
\text { Set }\end{array}$ & $\begin{array}{l}\text { Oligonucleotide } \\
\text { sequences }\left(5^{\prime} \rightarrow 3^{\prime}\right)\end{array}$ & $\begin{array}{l}\text { Tmem 16a } \\
\text { region }\end{array}$ & $\begin{array}{l}\text { Size } \\
(\mathrm{bp})\end{array}$ & $\begin{array}{c}\text { Alternative } \\
\text { exon(s) identified }\end{array}$ \\
\hline $\bar{A}$ & $\begin{array}{l}\text { ACAGGCGGCCACGATGAGGGTC (sense) } \\
\text { GAGCCTGCATCCAGCGACGCC (antisense) }\end{array}$ & Exons 1-2 & 348 & \\
\hline B & $\begin{array}{l}\text { AGGAGGGTGCAGCACAGCGA (sense) } \\
\text { ATTGGCCAGCAGGCTCGTGA (antisense) }\end{array}$ & Exons 2-7 & $\begin{array}{l}582 \\
648\end{array}$ & + Exon $6 b$ \\
\hline \multirow[t]{7}{*}{ C } & GAGAACGACGTGTACAAAGGCCAAG (sense) & Exons 6-16 & 714 & + Exon 13 \\
\hline & TTAGTGAGGTAGGCTGGGAACCGAT (antisense) & & 780 & + Exons $6 b+13$ \\
\hline & & & 792 & + Exons $13+15$ \\
\hline & & & 858 & + Exons $6 b+13+15$ \\
\hline & & & 579 & - Exons $10+13$ \\
\hline & & & 657 & - Exons $10+13+15$ \\
\hline & & & 708 & + Exons $6 b+13-14$ \\
\hline \multirow[t]{2}{*}{$\mathrm{D}$} & ACTGACAAAGTGAAGCTGACA (sense) & Exons $16-20$ & 526 & \\
\hline & TGTTCTGGATCAGCTGTTC (antisense) & & 414 & - Exon 18 \\
\hline \multirow[t]{2}{*}{ E } & TTTCCGTTCCTTCCGAATGG (sense) & Exons $19-23$ & 460 & \\
\hline & CTTCCCAATGCCTCTGAGGATA (antisense) & & & \\
\hline \multirow[t]{2}{*}{$\mathrm{F}$} & TGTCACTGAGCTCCGAAGGCCG (sense) & Exons 22-25 & 384 & \\
\hline & ACGATGACAAACGCCAGCCGG (antisense) & & & \\
\hline \multirow[t]{2}{*}{ G } & AGTAAGAACGGGACCATGCACGG (sense) & Exons $24-26$ & 509 & \\
\hline & CGCTGGCATAGCTACAGGACGC (antisense) & & & \\
\hline
\end{tabular}


and exclusion of exon 18 would result in a C-terminus truncated TMEM16A protein containing four membrane spanning domains (Figure 5A). Translation of mouse TMEM16A yields a protein of $\sim 110 \mathrm{kDa}$. The exclusion of exon 18 in the mouse transcript could result in a truncated TMEM16A protein of $\sim 60-75 \mathrm{kDa}$ (depending upon which other alternative exons reported in this study are also included or excluded). Interestingly, two independent studies have reported an additional signal on Western blots for TMEM16A $[25,36]$ which were much lower $(\sim 80 \mathrm{kDa})$ than the expected $110 \mathrm{kDa}$. From the results presented in this study, it is possible that the lower molecular weight band corresponds to an alternatively spliced transcript that encodes a truncated TMEM16A protein.

The $\Delta 3$ 'exon 1 and $\Delta 3$ 'exon 21 alternative donor sites are the result of wobble splicing occurring at donor splice sites located in close proximity, commonly known as tandem splice sites. Alternative splicing at tandem splice sites occurs frequently in many species and can result in subtle variations in transcripts and the proteins that they encode [37]. Use of the Tmem16a $\Delta 3$ 'exon 1 splice site, results in the exclusion of the last 3 nucleotides (GTG) of

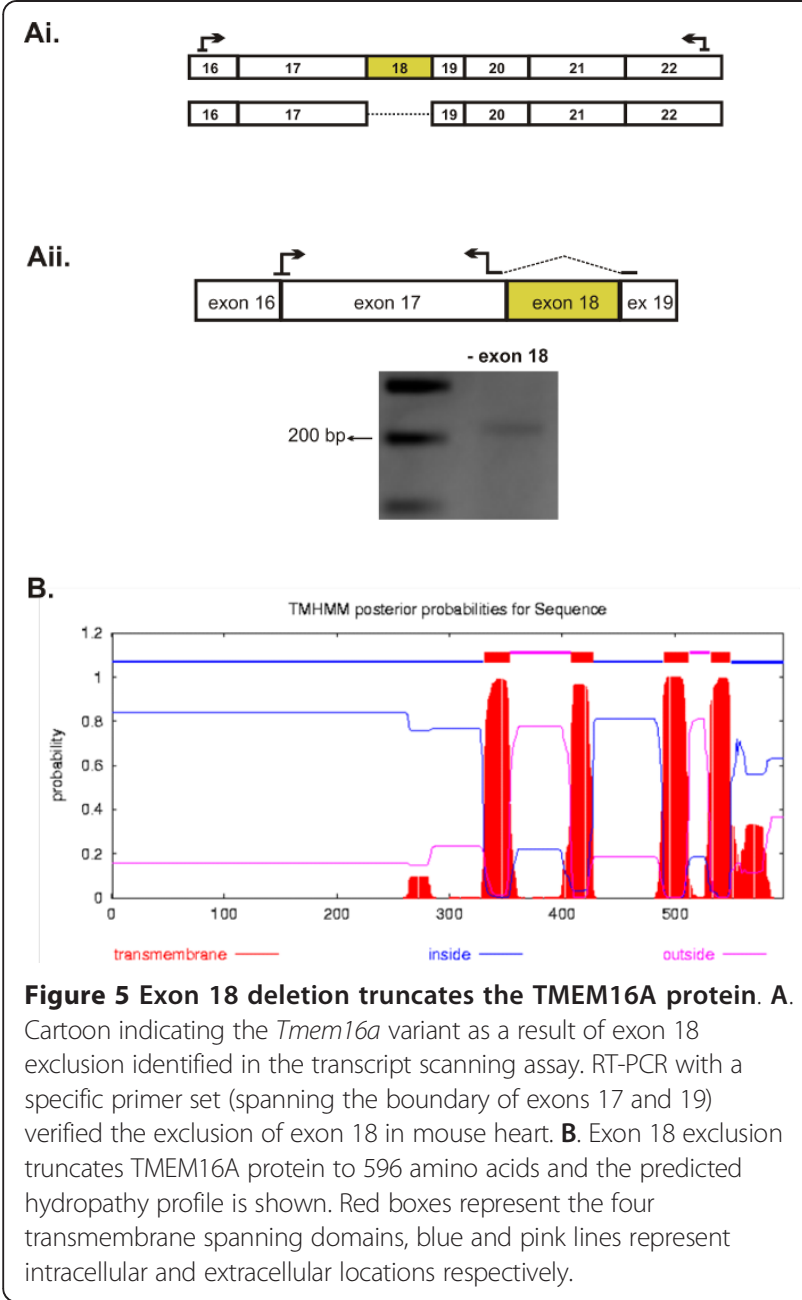

exon 1. The mouse Tmem16a genomic sequence was queried against the TassDB database of alternative tandem splice sites [38], and the $\Delta 3$ 'exon 1 was recognized as tandem splicing at donor splice site with the motif GYNGYN. Indeed, a BLASTn search of the EST database revealed 27 ESTs without this 3' GTG and two ESTs that contained it. This alternative splice event is not expected to alter TMEM16A protein since exon 1 is non-coding. Skipping of exon 1 and part of exon 2 has been reported in human TMEM16A [13], however, Ferrera et al failed to identify transcripts lacking this region [22] and considered that its splicing may be under the control of an alternative promoter and influenced by certain physiological or pathophysiological conditions. Indeed, a recent study showed that a novel TMEM16A variant with an alternative 5' end is highly expressed in gastric smooth muscle of patients with diabetic gastroparesis compared with controls [23]. Apart from $\Delta 3$ 'exon 1 tandem splice site, our study did not identify additional variation at the 5 'end. The $\Delta 3$ 'exon 21 tandem splice site would result in 4 additional (GTGA) nucleotides at the 3' end of exon 21 . This is predicted to shift the protein out of frame at amino acid M702 [GenBank: NP_848757], resulting in a truncated TMEM16A protein with an altered C-terminus of $\sim 84 \mathrm{kDa}$. According to TassDB2 [39] this tandem splice site is conserved in human, dog and chicken Tmem16a sequence.

\section{Distribution of novel Tmem 16a alternative splice variants in other tissues}

This study focused on identifying Tmem16a splice variants in heart. We extended this analysis to investigate if these novel variants are expressed in other mouse tissues from which a calcium-activated chloride current can be recorded. Initially, we confirmed Tmem16a expression in mouse brain, lung, stomach (antrum), small intestine, colon, kidney and bladder tissues (data not shown). All organs were dissected out with their attached vasculature. Total RNA obtained from each of these organs therefore contains a mixed population of cells. We then examined the expression of the following exons: $+6 \mathrm{~b},-10,+13,+13 \mathrm{~b}$, $-14,+15$ and -18 . The distribution of Tmem16a alternative exon variants in these mouse tissues is summarized (Table 4). In addition to heart, all of the tissues examined express the following variants; $+6 \mathrm{~b},-10,+13,+13 \mathrm{~b}$ and +15 . The -14 exon variant was only detected in heart, lung, small intestine and colon. The -18 exon variant was detected in all tissues examined apart from kidney. The molecular diversity of Tmem16a may contribute to numerous proteomic variations of TMEM16A channels that may finetune channel functions in specific tissue types.

\section{Conclusion}

This report provides details of Tmem16a genomic organization and our analyses have detected previously 
Table 4 Distribution of Tmem16a exon variants in mouse tissues

\begin{tabular}{|c|c|c|c|c|c|c|c|c|}
\hline & heart & brain & lung & stomach & s.int. & colon & kidney & bladder \\
\hline+ exon $6 b$ & ++ & +++ & +++ & +++ & +++ & +++ & +++ & + \\
\hline - exon 10 & + & + & + & + & + & + & + & + \\
\hline+ exon 13 & +++ & +++ & +++ & +++ & +++ & +++ & +++ & ++ \\
\hline+ exon $13 b$ & ++ & +++ & +++ & ++ & +++ & +++ & ++ & + \\
\hline - exon 14 & + & n.d. & + & n.d. & + & + & n.d. & n.d. \\
\hline+ exon 15 & ++ & ++ & + & +++ & +++ & +++ & +++ & + \\
\hline - exon 18 & + & + & + & + & +++ & +++ & n.d. & + \\
\hline
\end{tabular}

s.int. (small intestine) +++ indicates high, ++ moderate and + low expression. n.d., not detected.

unidentified alternative exons. We identified Tmem16a transcript variants consisting of different exon usage events involving exons $6 \mathrm{~b}, 10,13,14,15$ and 18. Many of these exons are expressed in various combinations. A number of other splicing events including a novel exon 13b, tandem splice sites of exon 1 and 21 and two intron retention events were also identified. The alternative splicing events indentified here indicate that a varied population of TMEM16A channels is expressed in mouse heart and other tissues. The heart is a highly heterogeneous tissue composed of numerous cell types tailored for specialized function. It is possible that many Tmem16a variants can be coexpressed in the same cells, or differentially distributed in distinct cell types within the myocardium. Determining the localization of different Tmem16a isoforms within tissues has important implications with regard to the function of Tmem16a. The functional consequence of TMEM16A channel diversity remains to be determined. We can speculate however, that TMEM16A variants would create channels with altered biophysical properties, such as that reported for exon 6b, 13 and 15 in TMEM16A [22,23]. It is also possible that regulation of the channels may be altered since several of these alternatively spliced exons contain phosphorylation sites and interacting domains, for example, PKC and CamKII (exon 18), PKA (exon 6b), Erk-D domain (exon 10) and a Src-SH3 domain (exon 15). TMEM16A channels have been shown to form homodimers [40,41], therefore, it is likely that TMEM16A variants may interact with each other to form functional channels in a similar manner to that reported for the hERG family [42]. Another possibility is that alternatively spliced isoforms may associate with additional accessory proteins. It is evident from this study that alternative splicing is an exquisite mechanism that operates to diversify TMEM16A channel structures by the combinatorial selection of alternatively spliced events. Future recombinant expression of different Tmem16a variants will reveal their physiological role.

\section{Additional material}

Additional file 1: Tables S1 and S2. TABLE S1: Primers used for RTPCR of Tmem16 paralogs. This table lists the GenBank accession number for Gapdh and Tmem16 paralogs, the oligonucleotide sequence of each primer and the expected amplicon sizes. TABLE S2: Primers used for RT-PCR of Tmem16a exon variants. This table lists the primer name, the oligonucleotide sequence of each primer, the annealing temperature of each primer set and the expected amplicon sizes.

Acknowledgements and Funding

The authors would also like to acknowledge Carly Gertler for excellent technical assistance. This project was supported by a NIH grant 1RO1HL091238 to FCB.

\section{Authors' contributions}

FCB and KEOD conceived the design of the study and prepared the manuscript. KEOD and RAP isolated RNA, constructed the libraries and prepared samples for sequencing. KEOD analyzed the sequencing data. All authors have read and approved the final manuscript.

Received: 23 March 2011 Accepted: 8 August 2011

Published: 8 August 2011

\section{References}

1. Black DL: Mechanisms of alternative pre-messenger RNA splicing. Annu Rev Biochem 2003, 72:291-336.

2. Britton FC, Wang GL, Huang ZM, Ye L, Horowitz B, Hume JR, Duan D: Functional characterization of novel alternatively spliced CIC-2 chloride channel variants in the heart. J Biol Chem 2005, 280:25871-25880.

3. Cunha SR, Le SS, Schott JJ, Mohler PJ: Exon organization and novel alternative splicing of the human ANK2 gene: implications for cardiac function and human cardiac disease. J Mol Cell Cardiol 2008, 45(6):724-734.

4. Dally S, Corvazier E, Bredoux R, Bobe R, Enouf J: Multiple and diverse coexpression, location, and regulation of additional SERCA2 and SERCA3 isoforms in nonfailing and failing human heart. J Mol Cell Cardiol 2010, 48(4):633-644.

5. George $\mathrm{CH}$, Rogers SA, Bertrand BM, Tunwell RE, Thomas NL, Steele DS, Cox EV, Pepper C, Hazeel CJ, Claycomb WC, Lai FA: Alternative splicing of ryanodine receptors modulates cardiomyocyte Ca2+ signaling and susceptibility to apoptosis. Circ Res 2007, 100(6):874-883.

6. Kupershmidt S, Snyders DJ, Raes A, Roden DM: A K+ channel splice variant common in human heart lacks a $\mathrm{C}$-terminal domain required for expression of rapidly activating delayed rectifier current. J Biol Chem 1998, 273(42):27231-27235

7. Liao P, Yong TF, Liang MC, Yue DT, Soong TW: Splicing for alternative structures of Cav1.2 Ca2+ channels in cardiac and smooth muscles. Cardiovasc Res 2005, 68(2):197-203.

8. Schroeter A, Walzik S, Blechschmidt S, Haufe V, Benndorf K, Zimmer T: Structure and function of splice variants of the cardiac voltage-gated sodium channel $\mathrm{Na}(\mathrm{v}) 1.5$. J Mol Cell Cardiol 2010, 49(1):16-24. 
9. Xu Y, Dong PH, Zhang Z, Ahmmed GU, Chiamvimonvat N: Presence of a calcium-activated chloride current in mouse ventricular myocytes. Am J Physiol Heart Circ Physiol 2002, 283:H302-H314.

10. Hiraoka M, Kawano S, Hirano Y, Furukawa T: Role of cardiac chloride currents in changes in action potential characteristics and arrhythmias. Cardiovasc Res 1998, 40:23-33.

11. Hume JR, Duan D, Collier ML, Yamazaki J, Horowitz B: Anion transport in heart. Physiol Rev 2000, 80:31-81.

12. Sorota S: Insights into the structure, distribution and function of the cardiac chloride channels. Cardiovasc Res 1999, 42:361-376

13. Caputo A, Caci E, Ferrera L, Pedemonte N, Barsanti C, Sondo E, Pfeffer U, Ravazzolo R, Zegarra-Moran O, Galietta LJ: TMEM16A, a membrane protein associated with calcium-dependent chloride channel activity. Science 2008, 322(5901):590-594

14. Schroeder BC, Cheng T, Jan YN, Jan LY: Expression cloning of TMEM16A as a calcium-activated chloride channel subunit. Cell 2008, 134:1019-1029.

15. Yang YD, Cho H, Koo JY, Tak MH, Cho Y, Shim WS, Park SP, Lee J, Lee B, Kim BM, Raouf R, Shin YK, Oh U: TMEM16A confers receptor-activated calcium-dependent chloride conductance. Nature 2008, 455(7217):1210-1215.

16. Pifferi $S$, Dibattista M, Menini A: TMEM16B induces chloride currents activated by calcium in mammalian cells. Pflugers Arch 2009, 458(6):1023-1038.

17. Schreiber R, Uliyakina I, Kongsuphol P, Warth R, Mirza M, Martins JR, Kunzelmann K: Expression and function of epithelial anoctamins. J Biol Chem 2010, 285(10):7838-7845

18. Stohr H, Heisig JB, Benz PM, Schoberl S, Milenkovic VM, Strauss O, Aartsen WM, Wijnholds J, Weber BH, Schulz HL: TMEM16B, a novel protein with calcium-dependent chloride channel activity, associates with a presynaptic protein complex in photoreceptor terminals. J Neurosci 2009, 29:6809-6818.

19. Hartzell C, Putzier I, Arreola J: Calcium-activated chloride channels. Annu Rev Physiol 2005, 67:719-758.

20. Huang X, Godfrey TE, Gooding WE, McCarty KS Jr, Gollin SM: Comprehensive genome and transcriptome analysis of the $11 q 13$ amplicon in human oral cancer and synteny to the 7F5 amplicon in murine oral carcinoma. Genes Chromosomes Cancer 2006, 45:1058-1069.

21. O'Driscoll KE, Hatton WJ, Britton FC: Alternative splicing of the murine Tmem16a transcript in heart. FASEB J 2010, 24:28.

22. Ferrera L, Caputo A, Ubby I, Bussani E, Zegarra-Moran O, Ravazzolo R, Pagani F, Galietta LJ: Regulation of TMEM16A chloride channel properties by alternative splicing. J Biol Chem 2009, 284(48):33360-33368.

23. Mazzone A, Bernard CE, Strege PR, Beyder A, Galietta LJ, Pasricha PJ, Rae JL, Parkman HP, Linden DR, Szurszewski JH, Ordog T, Gibbons SJ, Farrugia G: Altered expression of Ano1 variants in human diabetic gastroparesis. $J$ Biol Chem 2011, 286(15):13393-13403.

24. Hwang SJ, Blair PJ, Britton FC, O'Driscoll KE, Hennig G, Bayguinov YR, Rock JR, Harfe BD, Sanders KM, Ward SM: Expression of anoctamin 1/ TMEM16A by interstitial cells of Cajal is fundamental for slow wave activity in gastrointestinal muscles. J Physiol 2009, 587:4887-4904.

25. Davis AJ, Forrest AS, Jepps TA, Valencik ML, Wiwchar M, Singer CA, Sones WR, Greenwood IA, Leblanc N: Expression profile and protein translation of TMEM16A in murine smooth muscle. Am J Physio/ Cell Physiol 2010, 299(5):C948-C959.

26. Kozak M: Interpreting cDNA sequences: some insights from studies on translation. Mamm Genome 1996, 7:563-574.

27. Smith CW, Valcarcel J: Alternative pre-mRNA splicing: the logic of combinatorial control. Trends Biochem Sci 2000, 25(8):381-388.

28. Reed $R$, Maniatis $T$ : Intron sequences involved in lariat formation during pre-mRNA splicing. Cell 1985, 41(1):95-105.

29. Rautmann $G$, Breathnach $R$ : A role for branchpoints in splicing in vivo. Nature 1985, 315(6018):430-432

30. Coolidge CJ, Seely RJ, Patton JG: Functional analysis of the polypyrimidine tract in pre-mRNA splicing. Nucleic Acids Res 1997, 25(4):888-896.

31. Sakabe NJ, de Souza SJ: Sequence features responsible for intron retention in human. BMC Genomics 2007, 8:59.

32. Lareau LF, Green RE, Bhatnagar RS, Brenner SE: The evolving roles of alternative splicing. Curr Opin Struct Biol 2004, 14(3):273-282.

33. Bell TJ, Miyashiro KY, Sul JY, McCullough R, Buckley PT, Jochems J, Meaney DF, Haydon P, Cantor C, Parsons TD, Eberwine J: Cytoplasmic BK (Ca) channel intron-containing mRNAs contribute to the intrinsic excitability of hippocampal neurons. Proc Natl Acad Sci USA 2008, 105(6):1901-1906.
34. Bell TJ, Miyashiro KY, Sul JY, Buckley PT, Lee MT, McCullough R, Jochems J, Kim J, Cantor CR, Parsons TD, Eberwine JH: Intron retention facilitates splice variant diversity in calcium-activated big potassium channel populations. Proc Natl Acad Sci USA 2010, 107(49):21152-21157.

35. Hartzell HC, Yu K, Xiao Q, Chien LT, Qu Z: Anoctamin/TMEM16 family members are Ca2+-activated Cl- channels. J Physiol 2009, 587:2127-2139.

36. He Q, Halm ST, Zhang J, Halm DR: Activation of the basolateral membrane $\mathrm{Cl}$ - conductance essential for electrogenic $\mathrm{K}+$ secretion suppresses electrogenic Cl- secretion. Exp Physiol 2011, 96(3):305-316.

37. Hiller M, Platzer M: Widespread and subtle: alternative splicing at shortdistance tandem sites. Trends Genet 2008, 24(5):246-255.

38. Hiller M, Nikolajewa S, Huse K, Szafranski K, Rosenstiel P, Schuster S, Backofen R, Platzer M: TassDB: a database of alternative tandem splice sites. Nucleic Acids Res 2007, , 35 Database: D188-D192.

39. Sinha R, Lenser T, Jahn N, Gausmann U, Friedel S, Szafranski K, Huse K, Rosenstiel P, Hampe J, Schuster S, Hiller M, Backofen R, Platzer M: TassDB2 A comprehensive database of subtle alternative splicing events. $B M C$ Bioinformatics 2010, 11:216.

40. Fallah G, Romer T, Detro-Dassen S, Braam U, Markwardt F, Schmalzing G: TMEM16A(a)/anoctamin-1 shares a homodimeric architecture with CLC chloride channels. Mol Cell Proteomics 2011, 10(2):M110.

41. Sheridan JT, Worthington EN, Yu K, Gabriel SE, Hartzell HC, Tarran R: Characterization of the Oligomeric Structure of the Ca2+-activated ClChannel Ano1/TMEM16A. J Biol Chem 2011, 286(2):1381-1388.

42. Guasti L, Crociani O, Redaelli E, Pillozzi S, Polvani S, Masselli M, Mello T, Galli A, Amedei A, Wymore RS, Wanke E, Arcangeli A: Identification of a posttranslational mechanism for the regulation of hERG1 K+ channel expression and hERG1 current density in tumor cells. Mol Cell Biol 2008, 28(16):5043-5060.

doi:10.1186/1471-2199-12-35

Cite this article as: O'Driscoll et al:: Increased complexity of Tmem16al Anoctamin 1 transcript alternative splicing. BMC Molecular Biology 2011 12:35.

\section{Submit your next manuscript to BioMed Central and take full advantage of:}

- Convenient online submission

- Thorough peer review

- No space constraints or color figure charges

- Immediate publication on acceptance

- Inclusion in PubMed, CAS, Scopus and Google Scholar

- Research which is freely available for redistribution

Submit your manuscript at www.biomedcentral.com/submit
C Biomed Central 